\title{
AUDIT OF PERFORATION PERITONITIS IN RURAL SETUP AT NKPSIMS AND RC NAGPUR FOR 2010-2012
}

Satish Deshmukh, Rajiv K. Sonarkar, Dhananjay Pandey, Rahul Saboo, Pratik Shah

1. Associate Professor. Department of General Surgery, NKPSIMS and RC and LMH, Nagpur.

2. Assistant Professor. Department of General Surgery, NKPSIMS and RC and LMH, Nagpur.

3. Junior Resident. NKPSIMS and RC and LMH, Nagpur.

4. Junior Resident. NKPSIMS and RC and LMH, Nagpur.

5. Junior Resident. NKPSIMS and RC and LMH, Nagpur.

\section{CORRESPONDING AUTHOR:}

Dr. Rajiv K. Sonarkar,

Department of General Surgery,

NKPSIMS and RC and LMH,

Digdoh hills, Hingna road,Nagpur.

E-mail: drsrajiv@rediffmail.com

ABSTRACT: Fifty- nine patients who presented in the Casualty and O.P.D of department of General Surgery of NKPSIMS \& RC \&LMH with diagnosis of perforation peritonitis over a study period of one and half years were included. In most of the cases diagnosis was made by clinical examination supplemented by investigations in the form of standing X-ray chest PA view with domes of diaphragm, Ultrasound abdomen and blood test. After resuscitation Laparotomy was done in all the patients and thorough peritoneal lavage was done. A note of the site, size, type, number of perforations was made and biopsy was taken from the edge of the perforation. The most common cause of gastrointestinal perforation in our study was duodenal perforation. Primary closure of the perforation was most commonly done procedure. The overall mortality was $6 \%$. Morbidity like wound infection, fever, respiratory complications, residual abscess, dyselectrolytemia, burst abdomen, jaundice, sepsis, cardiac complications, and anastomotic disruption was studied.

KEY WORDS: Perforation Peritonitis, Duodenal Perforation, Laparotomy, Audit.

INTRODUCTION: Gastrointestinal perforation has been surgical problem since the time immortal. Scientists have found evidence of gastrointestinal perforations in Egyptian mummies. Perforation is said to occur once a pathology which extends through the full thickness of the hollow viscus leading to peritoneal contamination with intraluminal contents. Perforation can occur anywhere in the gastrointestinal tract starting from oesophagus to the rectum. Gastrointestinal perforation in our region generally occurs as a result of chronic inflammation due to Helicobacter pylori, NSAIDs like aspirin, stress, excessive smoking, alcohol, or coffee consumption. Other causes include appendicitis, diverticulitis, typhoid, malignancy. Instrumentation and blunt / penetrating abdominal trauma also account for a large number of cases of perforation peritonitis (1). Crohn's disease and less commonly ulcerative colitis are rare causes of perforation (2). If untreated, it leads to bacteremia, generalized sepsis, multiorgan failure, shock and abdominal abscess formation. The first successful surgical management for any gastrointestinal perforation was done for perforated gastric ulcer by Ludwig Heusner in Germany in 1892 in the form of partial gastrectomy (3). Gastrointestinal perforation is a serious surgical problem in developing nations with substantial morbidity and 
mortality and is one of the most common cause of emergency surgery performed in NKPSIMS \& RC AND LMH, DIGDOH HILLS, HINGNA ROAD NAGPUR.

MATERIALS AND METHODS: This was hospital based cross section retrospective study undertaken in Department of Surgery, NKPSIMS AND RC, DIGDOH HILLS, HINGNA ROAD NAGPUR from January 2010 to march 2012. Fifty-nine cases of gastrointestinal perforation reporting to emergency department were included in the study. All patients admitted under the study were put to detailed history taking including history of acid peptic disease, prolonged NSAIDS use, smoking, history of abdominal trauma and any other associated disease or related to cause of gastrointestinal perforation. A complete clinical examination was done. All patients were stabilized hemodynamically and broad spectrum antibiotics usually a combination of injectable third generation cephalosporin and metronidazole was administered. Blood transfusion was given whenever indicated. All the routine investigations were done, which included haemogram, blood grouping, kidney function tests, serum electrolytes, chest X-ray and electrocardiogram. Special investigations in the form of erect X-Ray abdomen with domes of diaphragm, Ultrasound abdomen were done. After confirmation of diagnosis of gastrointestinal perforation patients underwent emergency exploratory laparotomy through a midline incision. At the time of surgery, the source of contamination was sought for and appropriate procedure was performed. Further a note of the site of perforation, size of perforation, type of perforation, number of perforations, Amount of contamination was made. Biopsy was taken from the perforation edge whenever required. Every case was put to thorough normal saline peritoneal lavage. Abdominal drains were kept in every patient. All the patients were followed postoperatively by nothing per oral, nasogastric suction, intravenous fluids and antibiotic cover. Early ambulation was ensured and whenever required appropriate physiotherapy administered. In cases of moderate to severe anaemia, blood transfusion was given. Complications if occurred were vigorously managed. Patients were allowed oral diet after the return of bowel sounds, passage of flatus and /or stools. The patients were followed up in surgical OPD after discharge.

RESULTS: This was hospital based cross section retrospective study undertaken over a period of one and half years in the Department of Surgery, NKPSIMS \&RCAND LMH ,DIGDOH HILLS,HINGNA ROAD NAGPUR from 2011 - march 2012 and fifty-nine patients who underwent emergency laparotomy for gastrointestinal perforation were included in the study. Out of the total patients included in the study 54 were males and 5 were females. For observation and result of this study we used the CHI SQUERE STATISTICAL FORMUAE .Overall male to female ratio was 10.8:1 The group of patients studied appears to be a fair representative of the various demographic patterns associated with the disease. The majority of patients were in the third to sixth decade of life with the highest incidence in the fifth decade of life. Most common symptom at presentation was pain which was present in all the patients followed by vomiting in $(80 \%)$ abdominal distention in (76\%), fever in $(20 \%)$ constipation in $(14 \%)$ sepsis in $(8 \%)$ and shock in (6\%). In our study (26\%) cases had associated co- morbid conditions. Most common associated disease was COPD followed by hypertension, cardiac abnormalities, diabetes, and tuberculosis, deranged KFT'. Preoperative diagnosis was mainly clinical supplemented with investigations in the form of X-ray chest showing free gas under right dome of diaphragm, ultrasound of abdomen wherever indicated. Most of the patients $60 \%$ were operated within 24 
hours of perforation. 24\% patients were operated between 24-48 hours of perforation, $9 \%$ between 48-72 hours and the remaining $7 \%$ after 72 hours of perforation.

The most common cause of gastrointestinal perforation in our study was duodenal ulcer perforation (97\%), gastric perforations in (3\%), In cases of duodenal perforation all the perforations were in the anterior wall of the duodenum mostly in the first part. In cases of gastric perforations a biopsy from the edge of perforation was always taken and none of the patients had evidence of gastric malignancy in the biopsy. The most common performed procedure was primary closure of the perforation in all fifty-nine patients in the study.

The overall mortality observed in our study was (6\%). 1patients died intraoperatively and 1 patients died within 12 hours of surgery. 1patients died due to pulmonary or cardiac complication and septicemia. Common factors in all the deaths were late presentation, extremes of age, low preoperative haemoglobin, poor nutrition, , poor cardiac risk patients, irreversible shock, and septicemia and associated co-morbid conditions. The most common complication observed was wound infection which occurred in (16\%)of the patients followed by fever in $(8 \%)$ of the cases, respiratory complications in $(6 \%)$, residual abscesses in (5\%), burst abdomen in (0\%), dyselectrolemia in (4\%), sepsis in (3\%), cardiac complications in (3\%),

Table- 1. Age Distribution (n59)

\begin{tabular}{|l|l|l|}
\hline Age in Years & No. of patients & $\%$ \\
\hline$<10$ & 1 & $1.69 \%$ \\
\hline $11-20$ & 9 & $15.25 \%$ \\
\hline $21-30$ & 10 & $16.94 \%$ \\
\hline $31-40$ & 13 & $22.03 \%$ \\
\hline $41-50$ & 9 & $15.25 \%$ \\
\hline $51-60$ & 13 & $22.25 \%$ \\
\hline $61-70$ & 4 & $6.77 \%$ \\
\hline
\end{tabular}

Table-2. Etiology of Perforation ( $n=59)$

\begin{tabular}{|l|l|l|}
\hline Cause of perforation & No. of patients & \% \\
\hline Duodenal ulcer & 56 & $94.91 \%$ \\
\hline Gastric ulcer & 3 & $5.08 \%$ \\
\hline
\end{tabular}

Table-3. Sex Profile of Patients (n=59)

\begin{tabular}{|l|l|l|}
\hline Cause of perforation & Male & Female \\
\hline Duodenal ulcer & 51 & 5 \\
\hline Gastric ulcer & 3 & - \\
\hline
\end{tabular}


Table-4. Size of Perforation ( $n=59)$

\begin{tabular}{|c|c|}
\hline Size of perforation $\mathbf{( c m )}$ & No. of patients \\
\hline $0-<0.5 \mathrm{~cm}$ & 8 \\
\hline $0.5-<1 \mathrm{~cm}$ & 34 \\
\hline $1-<2 \mathrm{~cm}$ & 15 \\
\hline$>2 \mathrm{~cm}$ & 2 \\
\hline
\end{tabular}

Table-5. Complications Including Morbidity \& Mortality

\begin{tabular}{|l|l|l|}
\hline Complications & No. of Patients & \% \\
\hline Wound infection & 7 & $11.86 \%$ \\
\hline Fever & 12 & $20.33 \%$ \\
\hline Respiratory complications & 10 & $16.94 \%$ \\
\hline Residual abscess & 0 & $0 \%$ \\
\hline Dyselectrolytemia & 32 & $54.23 \%$ \\
\hline Burst abdomen & 1 & $1.69 \%$ \\
\hline Jaundice & 1 & $1.69 \%$ \\
\hline Sepsis & 10 & $16.94 \%$ \\
\hline Cardiac complications & 2 & $3.38 \%$ \\
\hline Anastomotic disruption & 0 & $0 \%$ \\
\hline Death & 4 & $6.77 \%$ \\
\hline
\end{tabular}

DISCUSSION: Perforation peritonitis is one of the most common surgical conditions encountered in surgical practice and is a common cause of morbidity and mortality and warrants early surgical intervention (4). Adequate resuscitation along with baseline investigations and broad spectrum antibiotics are imperative in each case. Further management depends upon the cause of peritonitis. In most of the cases the peritoneal contamination is caused by mixed flora both aerobic and anaerobic. Anatomical, pathological, and surgical factors may favour localization of peritonitis (5). However, in majority of the cases

peritonitis becomes diffuse when it occurs in patients with sudden anatomical disruption, extremes of age, immunodeficiency, perforation proximal to obstruction, stimulation of peristalsis and following trauma (6). The clinical presentation of the patients depends upon the site of perforation. Patients of duodenal perforation present with a short history of pain epigastrium or upper abdomen along with generalized tenderness and guarding $(4,7)$. In patients of diverticulitis patients are generally of old age and past history of constipation is present along with signs of peritonitis. Appendicular perforations have a characteristic pain starting in periumblical area or right iliac fossa along with vomiting and fever (8). There are also 
conspicuous signs present like guarding and rebound tenderness in right iliac fossa. Ileal perforations are usually preceded by a history of some medical disease followed by sudden onset of lower abdomen pain, vomiting, abdominal guarding and distention later on (9). In patients of trauma generalized peritoneal signs start developing after 2-3 hours of injury (2). In our study the most common cause of perforation was duodenal ulcer. Another study conducted by Gupta \& Kaushik shows the same result (10). Perforation of the proximal part of GIT were more common in our set up (11), which is in contrast to the studies from western countries where perforations are more common in the distal part (12). Perforation followed by acute appendicitis, enteric fever, trauma, obstruction, gastric ulcer perforation, malignancy, iatrogenic, recurrent in descending order. Malignancy is a rare cause of perforation peritonitis. It is seen only in $2 \%$ of our cases as compared to the western counterpart (13). Most common symptom at presentation was pain followed by vomiting, abdominal distention, fever and constipation. In our study we found that patients who presented early after perforation and had no associated co-morbid conditions behaved very well in the postoperative period. In patients with very poor general condition and irreversible shock, drains were put under local anaesthesia and adequate resuscitation along with antibiotic cover, blood transfusion was given to the patients and were taken up for laparotomy after their general condition improved. External drainage of the peritoneal cavity was made mandatory in every case by means of closed drainage system. The major complications which occurred following surgery included wound infection, fever, respiratory complications, residual abscesses in, burst abdomen, dyselectrolemia, jaundice, sepsis, cardiac complications, and anastomotic disruption which are known risk factors for high mortality (14). The overall mortality was $6 \%$. in few cases, multiple complications were seen in the same patient.

CONCLUSION: Perforation of the viscera is a common complication of acid peptic disease and typhoid. Acid peptic disease to some extent can be controlled by judicious use of NSAID as well as taking triple therapy for eradication of H-pylori. Early and primary treatment of typhoid should be undertaken on a grand scale and awareness among common practitioners should be cultivated.

\section{REFERENCE:}

1. Nadkarni F M, Shetly S D, Kagzi R S. Small-bowel perforation. A study of 32 cases. Archives Surgery 1981; 116: 53-57

2. Dorairajan L N, Gupta S, Deo S. V. Peritonitis in Indiadecade experience. Tropical Gastroenterology 1995;16: 33-38

3. Watkins RM, Dennison AR, Collin J. What has happened to perforated peptic ulcer? British J Surg 1984; 71:774-76.

4. Dean A, Clark C, Sinclair A. The late prognosis of perforated duodenal ulcer. Gut 1962; 3:60-64

5. Sharma L, Gupta S, Soin A S. Generalised peritonitis in India-tropical spectrum. Japan J Surg 1991; 21: 272-77

6. Bohen J, Boulanger M, Meakin L. Prognosis in generalized peritonitis in relation to cause and risk factors.Archives Surgery 1983; 118:285-87

7. Gupta S, Kaushik R, Sharma R .The management of large perforations of duodenal ulcers. BioMed Central Surgery2005; 5:1-9 
8. Mandava N, Kumar S, Walter F. Perforated colorectal carcinomas. Am J Surgery 1996; 172: $236-38$

9. Khanna AK and Misra Mk. Typhoid perforation of the gut. J Postgraduate Medicine1984; 60:523-25

10. Gupta S, Kaushik R. Peritonitis-The Eastern experience. World J Emerg Surg 2006; 1:13

11. Agarwall N, Saha S, Srivastava A, Chumber S, Dhar A, Garg S. Peritonitis 10 years experience in a single surgical unit. Trop Gastroenterol $2007,28(3): 117-20$.

12. Malangoni MA, Inui T. Peritonitis the western experience. World J Emerg Surg 2006 $1: 25$.

13. Di Venere B, Testini M, Miniello S, Piccinni G, Lissidini G, Carbone F, Bonomo GM. Rectal perforations. Personalexperience and literature review. Minerva Chir 2002 , 57(3):35762.

14. Uccheddu A, Floris G, Altana ML, Pisanu A, Cois A, Farci SL: Surgery for perforated peptic ulcer in the elderly.Evaluation of factors influencing prognosis Hepatogastroenterology $2003,50(54): 1956-68$. 